\title{
A fixed point theorem for deformation spaces of $G$-trees
}

\author{
Matt Clay
}

\begin{abstract}
For a finitely generated free group $F_{n}$, of rank at least 2, any finite subgroup of $\operatorname{Out}\left(F_{n}\right)$ can be realized as a group of automorphisms of a graph with fundamental group $F_{n}$. This result, known as $\operatorname{Out}\left(F_{n}\right)$ realization, was proved by Zimmermann, Culler and Khramtsov. This theorem is comparable to Nielsen realization as proved by Kerckhoff: for a closed surface with negative Euler characteristic, any finite subgroup of the mapping class group can be realized as a group of isometries of a hyperbolic surface. Both of these theorems have restatements in terms of fixed points of actions on spaces naturally associated to $\operatorname{Out}\left(F_{n}\right)$ and the mapping class group respectively. For a nonnegative integer $n$ we define a class of groups $(G V P(n))$ and prove a similar statement for their outer automorphism groups.
\end{abstract}

Mathematics Subject Classification (2000). Primary 20E08; secondary 20F65, 20F28.

Keywords. $G$-tree, deformation space, $\operatorname{Out}\left(F_{n}\right)$ realization, Nielsen realization.

Let $\Sigma$ be a closed surface with negative Euler characteristic. The mapping class group $\mathcal{M C} g(\Sigma)$ acts on Teichmüller space $T_{\Sigma}$, the space of hyperbolic metrics on $\Sigma$. A stabilizer in this action is an isometry group of some hyperbolic metric on $\Sigma$. Such groups must be finite. It is theorem of Kerckoff [14], known as Nielsen realization, that any finite subgroup of $\mathcal{M C} g(\Sigma)$ can be realized as a group of isometries for some hyperbolic metric on $\Sigma$. Therefore the finite subgroups of $\mathcal{M C} g(\Sigma)$ are exactly the subgroups with fixed points in $T_{\Sigma}$. In a similar manner, for a finitely generated free group $F_{n}$ of rank $n \geq 2$, the outer automorphism group $\operatorname{Out}\left(F_{n}\right)$ acts on Culler and Vogtmann's Outer space $X_{n}$ [7]. A stabilizer in this action is an isometry group of a some metric graph with fundamental group $F_{n}$. It is a theorem of Zimmermann [21], Culler [5] and Khramtsov [15], known as $\operatorname{Out}\left(F_{n}\right)$ realization, that any finite subgroup of $\operatorname{Out}\left(F_{n}\right)$ can be realized as a group of isometries of some metric graph with fundamental group $F_{n}$. Thus as for $\mathcal{M C g}(\Sigma)$ and $T_{\Sigma}$, the finite subgroups of $\operatorname{Out}\left(F_{n}\right)$ are exactly those subgroups with a fixed point in $X_{n}$.

For a nonnegative integer $n$ we introduce a class of groups denoted $G V P(n)$, and prove a similar realization statement for their outer automorphism groups. In other words, for every group $G \in G V P(n)$, there is a naturally associated space on which $\operatorname{Out}(G)$ acts and we are able to determine that certain subgroups of $\operatorname{Out}(G)$ 
related to stabilizers have a fixed point. For $n=0,1$ we show that any group that is commensurable to a subgroup of a stabilizer actually fixes a point (Corollary 4.2). The class $G V P(0)$ is the class of virtually finitely generated free groups of rank at least 2, thus our result is a generalization of $\operatorname{Out}\left(F_{n}\right)$ realization. In general, we are only able to show that subgroups of $\operatorname{Out}(G)$ commensurable to polycyclic subgroups of stabilizers fix a point.

We define $G V P_{0}(n)$ as the class of groups that act on locally finite simplicial trees without an invariant point or line, such that the edge stabilizers are virtually polycyclic subgroups of Hirsch length $n$. The subset of groups in $G V P_{0}(n)$ where this action is irreducible and cocompact is denoted $G V P(n)$. In the first section for any finitely generated group $G$ we describe topological spaces $\mathscr{D}$ on which certain subgroups of $\operatorname{Out}(G)$ act. These spaces are contractible in most cases. In particular, for $G \in G V P(n)$ we describe a contractible topological space $D_{G}$ on which the full group $\operatorname{Out}(G)$ acts. Our main theorem regarding this action is analogous to Nielsen realization and $\operatorname{Out}\left(F_{n}\right)$ realization.

Main Theorem. Suppose $G \in G V P(n)$ and $K$ is a polycyclic subgroup of $\operatorname{Out}(G)$ that fixes a point in $D_{G}$. If $H$ is a subgroup of $\operatorname{Out}(G)$ commensurable with $K$, then $H$ fixes a point in $\mathscr{D}_{G}$.

The proof of the above is similar to the proof for finite subgroups of $\operatorname{Out}\left(F_{n}\right)$. We review how to prove $\operatorname{Out}\left(F_{n}\right)$ realization. Starting with a finite subgroup $K$ of $\operatorname{Out}\left(F_{n}\right)$, lift this to the subgroup $\widetilde{K}$ in $\operatorname{Aut}\left(F_{n}\right)$. Then $\widetilde{K}$ is virtually free, hence $\widetilde{K}$ acts cocompactly on a simplicial tree $T$ with finite stabilizers by Stallings' theorem [20]. This induces a cocompact free action of $F_{n} \subseteq \widetilde{K}$ on $T$. Thus the finite group $K=\widetilde{K} / F_{n}$ acts on the quotient graph $T / F_{n}$, which represents a point in Outer space. Hence this point is fixed by $K$.

We seek to mimic this proof. The ingredient we will need is an analog to Stallings' theorem, i.e. we need to know when can we raise a splitting of a finite index subgroup to the whole group. For the special case we consider, this question has an answer due to Dunwoody and Roller [8]. We then show that any group that contains a finite index subgroup in $\operatorname{GVP}(n)$ is in fact itself in $G V P(n)$. Finally, if $G \in G V P(n)$ and $K$ is a polycyclic subgroup of $\operatorname{Out}(G)$ that stabilizes a point in $\mathscr{D}_{G}$, we show that $\widetilde{K}$, the lift of $K$ to $\operatorname{Aut}(G)$, is in $G V P\left(n^{\prime}\right)$ for some $n^{\prime}$, inducing an action of $G$. Thus we can proceed as above for $\operatorname{Out}\left(F_{n}\right)$.

Originally, we were only concerned only with a proof of realization for generalized Baumslag-Solitar (GBS) groups, the torsion-free groups in GVP(1). However, in doing so it became necessary to prove some statements in greater generality, which provided a proof for any $G V P(n)$-group.

Acknowledgements. I would like to thank my advisor Mladen Bestvina for suggesting this line of research and for his encouragement. Thanks are also due to Michah 
Sageev for discussions related to this work, to Gilbert Levitt for the correct references to $\operatorname{Out}\left(F_{n}\right)$ realization and to the referee for shorting an early version of this paper by pointing to Theorem 3.1 .

\section{Deformation spaces of $G$-trees}

For a finitely generated group $G$, a $G$-tree is a metric simplicial tree on which $G$ acts by isometries. We say two $G$-trees $T$ and $T^{\prime}$ are equivalent if there is a $G$ equivariant isometry between then. When we speak of a $G$-tree we will always mean the equivalence class of the $G$-tree. A subgroup is an elliptic subgroup of $T$ if it fixes a point in $T$. Given a $G$-tree there are two moves one can perform to the tree that do not change whether subgroups of $G$ are elliptic. These moves correspond to the isomorphism $A \cong A *_{C} C$ and are called collapse and expansion. For a detailed description of the moves see [10]. In [10] Forester proves the converse, namely if two cocompact $G$-trees have the same elliptic subgroups, then there is a finite sequence of collapses and expansions (called an elementary deformation) transforming one $G$-tree to the other.

We let $\mathcal{X}$ denote a maximal set of $G$-trees which are related by an elementary deformation. By the theorem of Forester mentioned above, an equivalent definition is as the set of all $G$-trees that have the same elliptic subgroups as some fixed $G$-tree. This set $\mathcal{X}$ is called an unnormalized deformation space. We will always assume that the $G$-trees are minimal and irreducible and that $G$ acts without inversions.

There is an action of $\mathbb{R}^{+}$on $\mathcal{X}$ by scaling, the quotient is called a deformation space and denoted $\mathscr{D}$. We [4] and independently Guirardel and Levitt [12], [13] have shown that for a finitely generated group, if the actions in $\mathscr{D}$ are irreducible and there is a reduced $G$-tree with finitely generated vertex stabilizers, then $\mathscr{D}$ is contractible. The topology for the preceding statement is the axes topology induced from the embedding $\mathscr{D} \rightarrow \mathbb{R P}^{\mathcal{C}}$ where $\mathcal{C}$ is the set of all conjugacy classes of elements in $G$, or equivalently the Gromov-Hausdorff topology. See [4] for details.

In general, the space $\mathscr{D}$ is acted on only by a subgroup of $\operatorname{Out}(G)$, where the action is precomposition. This subgroup is the subgroup of $\operatorname{Out}(G)$ that permutes the conjugacy classes of elliptic subgroups associated to $\mathscr{D}$.

If $G \in G V P(n)$ then there is a locally finite $G$-tree $T$ where all of the stabilizers are virtually polycyclic subgroups of Hirsch length $n$. We will show in the next section (Lemma 2.1) that the set of elliptic subgroups for this action is invariant under all automorphisms of $G$. Hence the deformation space containing $T$ is invariant under Out $(G)$. We denote this space as $\mathscr{D}_{G}$. Notice that if $G=F_{n}$, then $\mathscr{D}_{G}=X_{n}$, Culler and Vogtmann's Outer space. 


\section{Virtually polycyclic groups and the class of $G V P(n)$-groups}

A group $G$ which admits a filtration $\{1\}=G_{0} \triangleleft G_{1} \triangleleft \cdots \triangleleft G_{n}=G$ with $G_{i-1}$ normal in $G_{i}$ and $G_{i} / G_{i-1}$ cyclic is called polycyclic. The Hirsch length, denoted $\mathrm{h} G$, of a polycyclic group $G$ is the number of infinite cyclic factors in the above filtration. This is an invariant of $G$. If $G$ is polycyclic and $H$ is any finite index subgroup then $\mathrm{h} H=\mathrm{h} G$. In fact, if $H$ is a subgroup of $G$, then $\mathrm{h} H \leq \mathrm{h} G$ with equality if and only if $H$ has finite index in $G$. This allows us to define the Hirsch length of a group which contains a polycyclic group as a finite index subgroup. Such groups are called virtually polycyclic. These groups are also referred to as polycyclic-by-finite groups in the literature. Note that if $1 \rightarrow K \rightarrow G \rightarrow H \rightarrow 1$ is a short exact sequence then $H$ and $K$ are virtually polycyclic if and only if $G$ is. In this case, the Hirsch lengths satisfy h $G=\mathrm{h} H+\mathrm{h} K$.

As mentioned in the introduction, originally the main theorem was only intended for generalized Baumslag-Solitar groups. A group $G$ is a generalized BaumslagSolitar $(G B S)$ group if there is a cocompact $G$-tree where the stabilizer of any point is isomorphic to $\mathbb{Z}$. As the only $\mathbb{Z}$ subgroups of $\mathbb{Z}$ are necessarily of finite index, this $G$-tree must necessarily be locally finite. Equivalently, $G$ is a GBS group if it admits a graph of groups decomposition where all of the edge groups and vertex groups are isomorphic to $\mathbb{Z}$. Hence the nonelementary GBS groups (i.e. $G \neq \mathbb{Z}, \mathbb{Z} \oplus \mathbb{Z}$ or the Klein-bottle group) are the torsion-free groups in $G V P(1)$.

Such groups were first studied by Kropholler [16], where it is shown that GBS groups are the only finitely generated groups of cohomological dimension two that contain an infinite cyclic subgroup which intersects each of its conjugates in a finite index subgroup. It is clear that for a GBS group any vertex group in the above mentioned graph of groups decomposition satisfies this condition. Forester's Lemma 2.5 in [11] (a generalization of which appears as Lemma 2.1 below) implies that when the action does not have an invariant line, the elliptic subgroups are the only subgroups which satisfy this condition. As this condition is algebraic, the set of elliptic subgroups is invariant under all automorphisms of $G$, hence we can talk about an $\operatorname{Out}(G)$-invariant deformation space. We now generalize this fact to any $G V P(n)$-group.

Recall that a group is called slender if every subgroup is finitely generated. Virtually polycyclic groups are slender. Slenderness of a group $G$ is equivalent to every subgroup $H \subseteq G$ having property AR: whenever $H$ acts on a simplicial tree, $H$ either stabilizes a point or has an axis [9]. Throughout the following, we use the notation $H^{g}=g \mathrm{Hg}^{-1}$. We say two subgroups $H, H^{\prime}$ of $G$ are commensurable if $H \cap H^{\prime}$ has finite index in both $H$ and $H^{\prime}$. The commensurator of a subgroup $H \subseteq G$ is $\operatorname{Comm}_{G}(H)=\left\{g \in G \mid H\right.$ is commensurable with $\left.H^{g}\right\}$.

Lemma 2.1. (Forester [11]) Let $T$ be a locally finite $G$-tree such that the stabilizer of any point in $T$ is slender. If $T$ does not contain a $G$-invariant line, then a subgroup 
$H \subseteq G$ is elliptic if and only if $H$ is contained in a subgroup $K$, where $K$ is slender and $\operatorname{Comm}_{G}(K)=G$.

Proof. As $T$ is a locally finite simplicial tree, any vertex stabilizer is commensurable to all of its conjugates. Hence, if $H$ is elliptic, it is contained in a vertex stabilizer $K$ which satisfies the conclusion of the lemma.

For the converse suppose $H \subseteq G$ does not act elliptically and $H$ is contained in a slender subgroup $K$. Hence $K$ does not act elliptically. Let $L_{K}$ be its axis. Then the axis of $K^{g}$ is $g L_{K}$. If $K$ and $K^{g}$ are commensurable, then they have the same axis so $L_{K}=g L_{K}$. Hence if $K$ is commensurable to all of its conjugates, then $L_{K}$ is a $G$-invariant line.

Thus for such actions the elliptic subgroups are determined algebraically. In particular, the elliptic subgroups for these actions are invariant under $\operatorname{Aut}(G)$. When the action is cocompact, we can talk about an $\operatorname{Out}(G)$-invariant deformation space, denote this space $\mathscr{D}_{G}$. Hence every point in $\mathscr{D}_{G}$ is a locally finite $G$-tree where the stabilizers are virtually polycyclic of Hirsch length $n$. Since the $G$-trees in $\mathscr{D}_{G}$ are locally finite, $\mathscr{D}_{G}$ is a locally finite complex. Hence all of the stabilizers are commensurable. Our realization statement (Main Theorem) is a partial converse to this in the general case and the full converse if $n=0$ or 1 .

For $G \in G V P(n)$, as these actions are irreducible, $G$ contains a free subgroup of rank 2. Thus if $G$ acts on a tree $T$ with virtually polycyclic stabilizers of Hirsch length $n$, then $T$ cannot be a line. This will be used without further mention. We have another lemma essentially due to Forester about the splittings of $G V P(n)$-groups as amalgams over virtually polycyclic groups $K$ with $\mathrm{h} K=n$. Say that $G$ splits over $K$ if $G$ can either be written as a nontrivial free product with amalgamation $G=A *_{K} B$ or as an $\mathrm{HNN}$-extension $G=A *_{K}$.

Lemma 2.2 (Forester [11]). Suppose $G \in G V P(n)$ and $T \in \mathscr{D}_{G}$. If $G$ splits over a virtually polycyclic subgroup $K$ with $\mathrm{h} K=n$, then $K$ fixes a point in $T$. Moreover, the vertex group $(s)$ in this splitting are finitely presented and either $G V P_{0}(n)$-groups or virtually polycyclic with Hirsch length $n$ or $n+1$.

Proof. Let $Y$ be the Bass-Serre tree for the splitting of $G$ over $K$ and $H$ a vertex stabilizer for the $G$-tree $T$. Then similarly to Lemma 2.1, $H$ must act elliptically on $Y$ as $Y$ cannot contain a $G$-invariant line. Let $y \in Y$ be a vertex fixed by $H$ and $e$ an edge stabilized by $K$. There is some $g \in G$ such that $e$ separates $y$ from $g y$. As $H$ and $H^{g}$ are commensurable, there is a finite index subgroup $H^{\prime} \subseteq H$ stabilizing $e$, hence contained in $K$. As both $K$ and $H^{\prime}$ have Hirsch length $n, H^{\prime}$ has finite index in $K$, hence $K$ an $H$ are commensurable. Thus as $H$ fixes a point in any $G$-tree $T \in \mathscr{D}_{G}$, so does $K$. 
As for the moreover, suppose $A$ is a vertex group for the splitting of $G$ over $K$. We examine how $A$ acts on $T$. If $A$ fixes a point, then $A$ is virtually polycyclic with $\mathrm{h} A=n$. If $A$ has an invariant line on which it acts nontrivially, then there is a short exact sequence $1 \rightarrow K^{\prime} \rightarrow A \rightarrow \mathbb{Z} \rightarrow 1$ or $1 \rightarrow K^{\prime} \rightarrow A \rightarrow \mathbb{Z}_{2} * \mathbb{Z}_{2} \rightarrow 1$ where $K^{\prime}$ is commensurable to $K$. Hence $A$ is virtually polycyclic and h $A=n+1$. Otherwise this action implies that $A \in G V P_{0}(n)$.

To see that $A$ is finitely presented we can assume that $A \in G V P_{0}(n)$. As $K$ acts elliptically in $T$, using the action of $A$ on $T$ we can refine the splitting of $G$ over $K$ to get a graph of groups decomposition for $G$ that includes the graph of groups decomposition of $A$ with virtually polycyclic vertex and edge groups of Hirsch length $n$. As $G$ is finitely generated, after reducing we can assume that the graph of groups decomposition for $G$, hence the graph of groups decomposition of $A$, is a finite graph. Thus $A$ can be expressed as a finite graph of groups where all of the vertex and edge groups are virtually polycyclic of Hirsch length $n$. In particular, $A$ is finitely presented.

We record some properties about $G V P(n)$-groups that will be used in Section 4.

Lemma 2.3. Let $G \in G V P(n)$ then:

1. $\operatorname{cd}_{\mathbb{Q}} G=n+1$;

2. $G$ does not split over a virtually polycyclic group of Hirsch length less than n; and

3. the center of $G, Z(G)$, is a virtually polycyclic subgroup with $\mathrm{h} Z(G) \leq n$. The quotient $G / Z(G)$ is in $G V P\left(n^{\prime}\right)$ for $n^{\prime}=n-\mathrm{h} Z(G)$.

Proof. For 1. and 2. see Sections 6 and 7 in [3].

To see 3., let $T \in \mathscr{D}_{G}$. As the action is irreducible, $Z(G)$ must act trivially on $T$ [1]. Hence $Z(G)$ is a virtually polycyclic subgroup with $\mathrm{h} Z(G) \leq n$. Also, we have an induced irreducible cocompact action of $G / Z(G)$ on $T$, where the stabilizers are the quotients of the stabilizers for the $G$-action by $Z(G)$. Hence $G / Z(G)$ is in $G V P\left(n^{\prime}\right)$ where $n^{\prime}=n-\mathrm{h} Z(G)$.

Remark 2.4. As cohomological dimension is an invariant of the group, if $G \in$ $G V P(n)$, then $G \notin G V P\left(n^{\prime}\right)$ for $n \neq n^{\prime}$.

\section{Promoting finite index splittings}

The main step in proving $\operatorname{Out}\left(F_{n}\right)$ realization is to use Stallings' theorem to get a splitting of the virtually free subgroup of $\operatorname{Aut}\left(F_{n}\right)$ which is the lift of some finite subgroup in $\operatorname{Out}\left(F_{n}\right)$. In the present setting we will need an analog of Stallings' theorem 
to tell us when a splitting of a finite index subgroup $H \subseteq G$ over $K \subseteq H$ implies a splitting of the whole group $G$ over some subgroup $K^{\prime}$ which is commensurable to $K$. In general, we cannot expect a splitting of $G$. In our special case though, the answer is given by the following theorem of Dunwoody and Roller [8] as stated by Scott and Swarup [19]. The ends of the pair of groups $K \subseteq G$, denoted $e(G, K)$, is the number of ends of $\Gamma / K$ where $\Gamma$ is a Cayley graph for $G$. If $G$ splits over a subgroup $K$, then $e(G, K)>1$. See [19] or [20] for these notions.

Theorem 3.1 (Dunwoody-Roller [8] as stated in [19]). If $G, K$ are finitely generated subgroups with $e(G, K)>1$ and if $\operatorname{Comm}_{G}(K)=G$, then $G$ splits over a subgroup commensurable to $K$.

We can now prove our analog to Stallings' theorem.

Theorem 3.2. Let $G$ be a finitely presented group which has a finite index subgroup $H \in G V P(n)$, then $G \in G V P(n)$.

Proof. If $n=0$ then this is Stallings' theorem [20], so we assume that $n \geq 1$.

Let $T \in \mathscr{D}_{H}$ and $K$ be an edge stabilizer of $T$. As finite index subgroups of $H$ are in $G V P(n)$, we can assume that $H$ is normal in $G$. By Lemma 2.1, elliptic subgroups of $H$ are invariant under automorphisms of $H$, hence $\operatorname{Comm}_{G}(K)=G$. As $e(H, K)>1$ and $H$ is a finite index subgroup of $G$, we must have that $e(G, K)>1$. Then by Theorem 3.1, $G$ splits over a subgroup $K^{\prime}$ commensurable with $K$. Let $T^{\prime}$ be the Bass-Serre tree for this splitting of $G$ over $K^{\prime}$. If $T^{\prime}$ is locally finite then we are done as the vertex and edge stabilizers for $G$ acting on this tree are then commensurable to $K^{\prime}$ hence virtually polycyclic subgroups of Hirsch length $n$, therefore $G \in G V P(n)$.

Suppose that $T^{\prime}$ is not locally finite, we now show that we can split a vertex group for the graph of group decomposition induced by $H$ acting on $T^{\prime}$. As $T^{\prime}$ is not locally finite there is a vertex group $H_{v}$ which is not a virtually polycyclic subgroup of Hirsch length at most $n$. Suppose $G_{v}$ is the vertex group under the $G$-action, thus $H_{v}$ has finite index in $G_{v}$. As the induced action of $H_{v}$ on $T$ is nontrivial, we get a graph of groups decomposition for $H_{v}$. Then we can collapse this graph of groups decomposition to get a splitting of $H_{v}$ over some edge stabilizer $K_{v}$. Denote the Bass-Serre tree for this splitting as $T_{v}$. As $K_{v}$ is commensurable to $K$ we have $\operatorname{Comm}_{G}\left(K_{v}\right)=G$, thus $\operatorname{Comm}_{G_{v}}\left(K_{v}\right)=G_{v}$. As $H_{v}$ is finitely generated, $G_{v}$ is also. Therefore by as above by Theorem 3.1, $G_{v}$ splits as an amalgam over $K_{v}^{\prime}$ which is commensurable to $K_{v}$ hence also $K^{\prime}$.

As $K_{v}^{\prime}$ and $K^{\prime}$ are commensurable, in the Bass-Serre tree associated to the splitting of $G_{v}$ over $K_{v}^{\prime}, K^{\prime}$ acts elliptically. This allows us to refine the one edge splitting of $G$ over $K^{\prime}$ to get a two edge splitting of $G$ over $K^{\prime}$ and $K_{v}^{\prime}$. Once again, we have a 
Bass-Serre tree $T_{0}$ associated to this graph of groups decomposition, and the action of $H$ on $T_{0}$ induces a graph of groups decomposition for $H$.

If $T_{0}$ is not locally finite, repeat. As long as the resulting Bass-Serre tree is not locally finite we can continue. Since at each step, we add one edge to the quotient graph of groups decomposition of $G$, this process must terminate by Bestvina-Feighn [2].

We also note that recently Kropholler has proved a more general statement [17].

\section{Realization}

In this section we prove the main theorem. For the remainder of this paper, we let $G \in G V P(n)$ be fixed and $\mathscr{D}_{G}$ denote the $\operatorname{Out}(G)$-invariant deformation space discussed in Section 2.

Suppose that $K$ is a subgroup of $\operatorname{Out}(G)$ and $K$ fixes some point $T \in D_{G}$. Then $\widetilde{K}$ the lift of $K$ to $\operatorname{Aut}(G)$ consists of automorphisms $\phi$ such that there exists an isometry $h_{\phi}: T \rightarrow T$ where $h_{\phi}(g x)=\phi(g) h_{\phi}(x)$ for all $x \in T, g \in G$. As the $G$-trees in $\mathcal{D}_{G}$ are irreducible and minimal, $h_{\phi}$ is unique [6]. Thus we get a homomorphism $\widetilde{K} \rightarrow \operatorname{Isom}(T)$, i.e. $T$ is a $\widetilde{K}$-tree. It is easy to check that $\widetilde{K}$ extends the action of $G / Z(G)$ on $T$.

As $T$ is a locally finite tree, if the edge groups are virtually polycyclic then $\widetilde{K} \in G V P\left(n^{\prime}\right)$ for some $n^{\prime}$. In this case Theorem 3.2 implies that whenever $\widetilde{K}$ is a finite index subgroup of some group $\widetilde{H}$, then $\widetilde{H} \in G V P\left(n^{\prime}\right)$. Thus we have a $G$-tree fixed by $H$, the image of $\widetilde{H}$ in $\operatorname{Out}(G)$. We compute the edge stabilizers for the action of $\widetilde{K}$ on $T$ via the following sequences. For an edge $f \subseteq T$ denote by $G_{f}$ (respectively $\widetilde{K}_{f}$ ) the edge stabilizer of $f$.

Lemma 4.1. The edge stabilizers of $T$ for the $\widetilde{K}$-action, $\widetilde{K}_{f}$, fit into short exact sequences:

$$
1 \longrightarrow G_{f} / Z(G) \longrightarrow \widetilde{K}_{f} \longrightarrow K_{f} \longrightarrow 1
$$

where $K_{f}$ is the image of $\widetilde{K}_{f}$ in $K$. In particular, if $K_{f}$ is virtually polycyclic then $\widetilde{K}_{f}$ is a virtually polycyclic subgroup of $\widetilde{K}$.

Proof. This only place where exactness needs to be checked is that $G_{f} / Z(G)$ is the kernel of the map $\widetilde{K}_{f} \rightarrow K_{f}$. This follows as $\widetilde{K}$ extends the action of $G / Z(G)$, hence $G / Z(G) \cap \widetilde{K}_{f}=G_{f} / Z(G)$.

We can now prove the main theorem. 
Main Theorem. Suppose $G \in G V P(n)$ and $K$ is a polycyclic subgroup of $\operatorname{Out}(G)$ that fixes a point in $D_{G}$. If $H$ is a subgroup of $\operatorname{Out}(G)$ commensurable with $K$, then $H$ fixes a point in $\mathscr{D}_{G}$.

Proof. Suppose that $H \subseteq \operatorname{Out}(G)$ contains $K$ as a finite index subgroup where $K$ is polycyclic and fixes a point in $\mathscr{D}_{G}$. We then have the following short exact sequences:

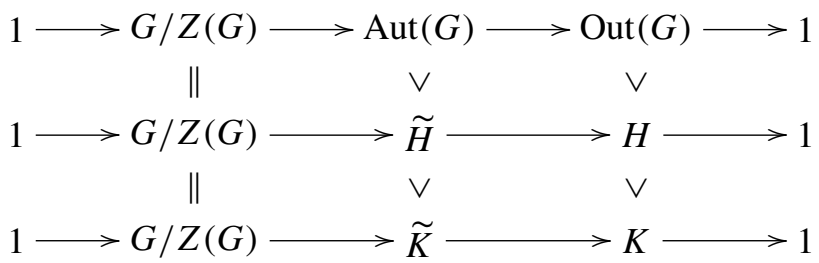

Then as $\widetilde{K} \in G V P\left(n^{\prime}\right)$ by Lemma 4.1, and as $\widetilde{H}$ contains $\widetilde{K}$ as a finite index subgroup, we have $\widetilde{H} \in G V P\left(n^{\prime}\right)$ by Theorem 3.2. Thus $\widetilde{H}$ acts on a locally finite tree $T^{\prime}$ inducing an action of $G / Z(G)$, hence also $G$, on $T^{\prime}$ with virtually polycyclic stabilizers necessarily of Hirsch length $n$ by Lemma 2.3. Let $T$ be the minimal subtree of $T^{\prime}$ for $G$. Then $T \in \mathscr{D}_{G}$ and clearly $H$ fixes this $G$-tree.

For $n=0$ or 1 , if $G \in G V P(n)$ then for any point $T \in D_{G}$ the vertex and edge groups have finite outer automorphism groups. Levitt [18] has shown that for these $G$-trees the stabilizer is virtually finitely generated abelian, hence we have the following corollary:

Corollary 4.2. If $G \in G V P(n)$ for $n=0$ or 1 and $H$ is a subgroup of $\operatorname{Out}(G)$ which contains a finite index subgroup that fixes a point in $\mathscr{D}_{G}$, then $H$ fixes a point in $\mathscr{D}_{G}$.

\section{References}

[1] H. Bass, R. Jiang, Automorphism groups of tree actions and of graphs of groups. J. Pure Appl. Algebra 112 (1996), 109-155. Zbl 0862.20021 MR 1402782

[2] M. Bestvina, M. Feighn, Bounding the complexity of simplicial group actions in trees. Invent. Math. 103 (1991), 449-469. Zbl 0724.20019 MR 1091614

[3] R. Bieri, Homological dimension of discrete groups. Queen Mary College Math. Notes, London 1976. Zbl 0357.20027 MR 0466344

[4] M. Clay, Contractibility of deformation spaces of G-trees. Alg. Geom. Topol. 5 (2005), 1481-1503. Zbl 02221903 MR 2186106

[5] M. Culler, Finite groups of outer automorphisms of a free group. In Contributions to Group Theory, Contemp. Math. 33, Amer. Math. Soc., Providence, RI, 1984, 197-207. Zbl 0552.20024 MR 0767107 
[6] M. Culler, J. Morgan, Group actions on $\mathbb{R}$-trees. Proc. London Math. Soc. (3) 55 (1987), 571-604. Zbl 0658.20021 MR 0907233

[7] M. Culler, K. Vogtmann, Moduli of graphs and automorphisms of free groups. Invent. Math. 84 (1986), 91-119. Zbl 0589.20022 MR 0830040

[8] M. J. Dunwoody, M. Roller, Splitting groups over polycyclic-by-finite subgroups. Bull. London Math. Soc. 25 (1993), 29-36. Zbl 0737.20012 MR 1190360

[9] M. J. Dunwoody, M. Sageev, JSJ-splittings for finitely presented groups over slender groups. Invent. Math. 135 (1999), 25-44. Zbl 0939.20047 MR 1664694

[10] M. Forester, Deformation and rigidity of simplicial group actions on trees. Geom. Topol. 6 (2002), 219-267. Zbl 02062447 MR 1914569

[11] M Forester, On the uniqueness of JSJ decompositions of finitely generated groups. Comment. Math. Helv. 78 (2003), 740-751. Zbl 1040.20032 MR 2016693

[12] V. Guirardel, G. Levitt, A general construction of JSJ splittings. Research announcement.

[13] V. Guirardel, G. Levitt, Outer space of a free product. Preprint, 2006. arXiv:math.GR/ 0501288.

[14] S. Kerckhoff, The Nielsen Realization Problem. Ann. of Math. 117 (1983), 235-265. Zbl 0528.57008 MR 0690845

[15] D. G. Khramtsov, Finite groups of automorphisms of free groups. Mat. Zametki 38 (1985), 386-392; English transl. Math. Notes 38 (1985), 721-724. Zbl 0595.20036 MR 0811572

[16] P. Kropholler, Baumslag-Solitar groups and some other groups of cohomological dimension two. Comment. Math. Helv. 65 (1990), 547-558. Zbl 0744.20044 MR 1078097

[17] P. Kropholler, A generalization of the Lyndon-Hochschild-Serre spectral sequence with applications to group cohomology and decompositions of groups. J. Group Theory 9 (2006), 1-25. Zbl 05042647 MR 2195835

[18] G. Levitt, Automorphisms of hyperbolic groups and graphs of groups. Geom. Dedicata 114 (2005), 49-70. Zbl 02227554 MR 2174093

[19] P. Scott, G. A. Swarup, Splittings of group and intersection numbers. Geom. Topol. 4 (2000), 179-218. Zbl 0983.20024 MR 1772808

[20] G. P. Scott, C. T. C. Wall, Topological methods in group theory. In Homological Group Theory (C. T. C. Wall, ed.), Lecture Notes, vol. 36, London Math. Soc. (1979), 137-203. Zbl 0423.20023 MR 0564422

[21] B. Zimmermann, Über Homöomorphismen $n$-dimensionaler Henkelkörper und endliche Erweiterungen von Schottky-Gruppen. Comment. Math. Helv. 56 (1981), 474-486. Zbl 0475.57015 MR 0639363

Received February 2, 2005; revised October 10, 2005

Matt Clay, Department of Mathematics, University of Oklahoma, Norman, OK 73019-0315, U.S.A.

E-mail: mclay@math.ou.edu 